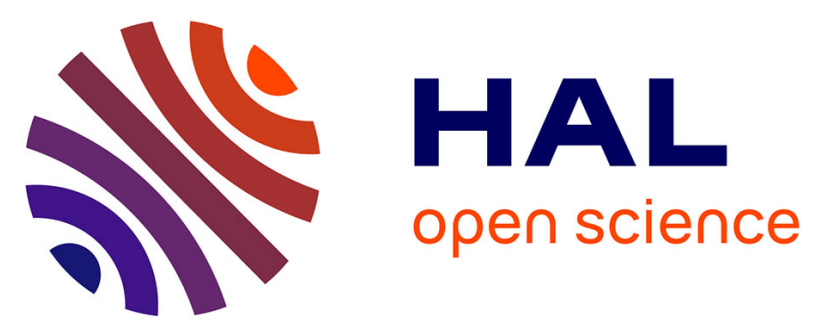

\title{
Study of the droplet size effect coupled with the laser light scattering in sprays for two-color LIF thermometry measurements
}

Alexandre Labergue, Alain Delconte, Guillaume Castanet, Fabrice Lemoine

\section{- To cite this version:}

Alexandre Labergue, Alain Delconte, Guillaume Castanet, Fabrice Lemoine. Study of the droplet size effect coupled with the laser light scattering in sprays for two-color LIF thermometry measurements. Experiments in Fluids, 2012, 52 (5), pp.1121 - 1132. 10.1007/s00348-011-1242-8 . hal-01570440

\section{HAL Id: hal-01570440 \\ https://hal.univ-lorraine.fr/hal-01570440}

Submitted on 30 Jul 2017

HAL is a multi-disciplinary open access archive for the deposit and dissemination of scientific research documents, whether they are published or not. The documents may come from teaching and research institutions in France or abroad, or from public or private research centers.
L'archive ouverte pluridisciplinaire HAL, est destinée au dépôt et à la diffusion de documents scientifiques de niveau recherche, publiés ou non, émanant des établissements d'enseignement et de recherche français ou étrangers, des laboratoires publics ou privés.

\section{(1)(1) $\$(0)$}

Distributed under a Creative Commons Attribution - NonCommercial - ShareAlikel 4.0 


\title{
Study of the droplet size effect coupled with the laser light scattering in sprays for two-color LIF thermometry measurements
}

\author{
A. Labergue $\cdot$ A. Delconte $\cdot$ G. Castanet . \\ F. Lemoine
}

\begin{abstract}
The present paper deals with a new optical diagnostic that aims to perform droplet temperature measurements based on the two-color laser-induced fluorescence (2cLIF). The 2 cLIF requires a single tracer, and the fluorescence intensity is collected on two spectral bands. The ratio of both intensities measured in the case of single monodisperse droplets depends in principle only on the temperature. However, the application in the case of polydispere sprays failed. Indeed, a dependence of the droplet size correlated to the depth of field of the optics, used for fluorescence collection, induces a bias in the ratio value. This work analyses these coupled effects by using combined LIF and Phase Doppler Analyser measurements. This device allows achieving fluorescence ratio measurements per droplet size class. The study is conducted with two different sprays in terms of particles size and density. The use of a third spectral band of detection (3cLIF) and a long distance microscope leads to correct the size effect and reduce the depth of field effect, respectively. These investigations are then demonstrated by measuring temperatures in an overheated water spray.
\end{abstract}

\section{Introduction}

Heat and mass transfer between droplets and gas in sprays are central in many engineering applications like internal combustion engines (Sazhin 2006; Maqua et al. 2008; Depredurand et al. 2011), spray cooling of hot surfaces (Castanet et al. 2009; Bernardin et al. 1997), or spray

A. Labergue $(\varangle) \cdot$ A. Delconte $\cdot$ G. Castanet $\cdot$ F. Lemoine LEMTA, Nancy-Université, CNRS 2, Avenue de la forêt de Haye, BP 160, 54504 Vandoeuvre-lès-Nancy, France e-mail: alexandre.labergue@ensem.inpl-nancy.fr drying. The optimization of these applications requires advanced physical models, and the temperature of the liquid phase is one of the most relevant parameters for the assessment of these models, although its measurement remains quite difficult.

Only few techniques are able to measure the droplet temperature in a spray. The global rainbow thermometry (GRT) is an extension of the standard rainbow technique that allows quantifying the refractive index of the liquid, and hence its temperature based on the angular position of the rainbow created by the laser scattering by the droplets. An interesting feature of the GRT relates to the smoothing of the perturbations induced by non-spherical droplets. In contrast, non-spherical droplets can lead to substantial errors with the standard rainbow technique dealing exclusively with single droplets (Vetrano et al. 2006; Van Beek et al. 2003).

Other techniques are based on laser-induced fluorescence and require introducing a tracer into the liquid to be sprayed. Temperature was measured using the thermal dependence of the florescence of excited-state complex (exciplex) produced by the association of an excited molecule (excimer) and a ground-state molecule (monomer) (Murray and Melton 1985; Gossage and Melton 1987). One of the main drawbacks of this method is its high quenching by oxygen, which may require operating in an oxygen-free medium.

In other fluorescence techniques, two fluorescent tracers are dissolved in the liquid (Sakakibara et al. 1997; Sakakibara and Adrian 1999; Kim et al. 2003). The fluorescence is collected on two spectral bands being part of the emission spectrum of the dyes. The ratio of the band signals varies with the liquid temperature and does not depend on the dimension of the measurement volume and the excitation power density. So far, this method has been 
reported in single-phase flows, and to the best of our knowledge, its implementation to evaporating droplets was never attempted.

The two-color laser-induced fluorescence ( $2 \mathrm{cLIF}$ ) technique used in this study is also a ratiometric method, but contrary to the technique described previously, it involves a single tracer. In principles, the ratio of the fluorescence signal measured on two spectral bands depends exclusively on the temperature; parameters like the tracer concentration, the probe volume, and the laser power density are eliminated in the ratio. The technique was successfully applied to measure the temperature of single evaporating or combusting droplets (Lavieille et al. 2001; Castanet et al. 2003), but its extension in sprays is not straightforward. In a recent paper, Labergue et al. (2010) pointed out that the spectral distribution of the detected fluorescence intensity was modified in a spray compared to a cell. Their results concerning calibrated monodisperse droplets revealed that the fluorescence ratio, in addition to be temperature sensitive, is also affected by a residual dependence on the droplet diameter. Similar modifications of the fluorescence spectrum due to the size of droplets were already observed by Habuchi et al. (2000) for droplets made of ethylene glycol/water mixtures and seeded with rhodamine $6 \mathrm{G}$. The phenomenon was attributed to dimer formation influenced by the radius of curvature of the droplet surface. The deviation of fluorescence ratio induced by this effect is all the more important that the droplet diameter is small. Obviously, in polydispersed spray, it is essential to account for this size effect or to circumvent it as much as possible.

This paper presents new investigations of combined size-temperature measurements by coupling the Phase Doppler Analysis (PDA) and the 2cLIF technique. Depredurand et al. (2011) recently described this coupling that allows obtaining correlations between the size and the temperature of the droplets by synchronizing the size measurements by PDA and the droplet temperature measurements by $2 \mathrm{cLIF}$. The aim of the present study is to investigate how these combined measurements are affected by the spray density and the polydispersion of the droplets in size, given the effect of the droplet size on the fluorescence and the depth of field of the receiving optics.

\section{Overview of the two-colors LIF thermometry}

\subsection{Principles of the two-colors LIF thermometry}

A comprehensive survey of the technique can be found in previous publications (Castanet et al. 2003; Lavieille et al. 2001). The liquid is preliminary seeded by a low concentration of a fluorescent tracer (sulforhodamine B also named Kiton red in the present case), which is temperature sensitive. The fluorescence of this tracer is induced by a laser (here the green line of an argon ion laser, $\lambda=514.5 \mathrm{~nm}$ ). The fluorescence spectrum is broadband and has a temperature-dependant shape. The fluorescence intensity $I_{f i}$ integrated on a spectral band $\left[\lambda_{i 1} ; \lambda_{i 2}\right]$ is expressed by (Lavieille et al. 2001):

$I_{f i}(T)=K_{\text {spec }, i} V_{c} I_{0} C f_{i}(T)$

where $I_{0}$ is the laser excitation intensity, $C$ the tracer concentration, $T$ the absolute temperature, and $V_{c}$ the collection volume of the fluorescence photons. This later is defined as the intersection of the illuminated part of the droplet volume and the field of view of the fluorescence collection optics. $K_{\mathrm{opt}, i}$ and $K_{\mathrm{spec}, i}$ are parameters influenced by the optical layout and the fluorescence properties of the tracers. These parameters are, therefore, constant during the measurements. The function $f_{i}(T)$ describes the temperature dependence of the fluorescence on the spectral band $i$ and it can be generally approximated by (Castanet et al. 2003):

$f i(T) \approx e^{\frac{a_{i}}{T^{2}}+\frac{b_{i}}{T}}$

where $a_{i}$ and $b_{i}$ are two coefficients that characterize the temperature sensitivity of the fluorescence signal on the spectral band $i$. To measure the temperature of moving droplets, the unknown parameters $C, V_{c}$ and $I_{0}$ must be removed. This can be achieved by collecting simultaneously the fluorescence signal on two spectral bands, respectively $I_{f 1}$ and $I_{f 2}$ and by calculating their ratio $R_{12}$ :

$R_{12}=\frac{I_{f 1}}{I_{f 2}}=\frac{K_{\mathrm{opt}, 1} K_{\mathrm{spec}, 1} f_{1}(T)}{K_{\mathrm{opt}, 2} K_{\mathrm{spec}, 2} f_{2}(T)}=\frac{K_{\mathrm{opt}, 1} K_{\mathrm{spec}, 1}}{K_{\mathrm{opt}, 2} K_{\mathrm{spec}, 2}} e^{\frac{a_{12}}{T^{2}}+\frac{b_{12}}{T}}$

where $a_{12}$ and $b_{12}$ denote respectively $a_{1}-a_{2}$ and $b_{1}-$ $b_{2}$. The ratio $R_{12}$ appears to solely dependent on the temperature. In the present study, the liquid is deionised water seeded by sulforhodamine B at a molar concentration $C=5 \times 10^{-6} \mathrm{~mol} \mathrm{l}^{-1}$ and the selected spectral bands are:

Band 1: [535, $545 \mathrm{~nm}]$

Band 2: $[615,750 \mathrm{~nm}]$.

These bands allow obtaining a high sensitivity of the fluorescence ratio to the temperature. A calibration is performed in a controlled temperature cell, made in borosilicate glass, with the same set-up as described in Depredurand et al. (2008). It gives $a_{12}=464,000 \mathrm{~K}^{2}$ and $b_{12}=-3,720 \mathrm{~K}$, which corresponds to a temperature sensitivity of about $0.9 \% / \mathrm{K}$. The uncertainties on the calibration procedure are given by performing several independent calibrations. Comparisons between them indicate that, in the worst scenario, the uncertainty induced by the calibration procedure is on the order of $1.5 \%$ on the fluorescence ratio at a given temperature, within the investigated temperature range. 
Finally, a single reference $R^{120}$ measurement at a known temperature $T_{0}$ allows eliminating both constants $K_{\text {opt, } i}$ and $K_{\text {spec, } i}$ in Eq. 3 . The normalized ratio $R_{12} / R_{120}$ is given by:

$\frac{R_{12}}{R_{120}}=\frac{f_{12}(T)}{f_{12}\left(T_{0}\right)}$

where $f_{12}(T)$ denotes $f_{1}(T)-f_{2}(T)$.

\subsection{Influence of the droplet Size}

A recent study has highlighted that the fluorescence ratio presents a dependence to the droplet diameter that is not included in Eq. 3. This effect corresponds to a significant distortion of the fluorescence spectrum. The deviation from the previous model is more important when the droplets are small, typically under $100 \mu \mathrm{m}$ (Labergue et al. 2010). This effect was reported for two fluorescent tracers (sulforhodamine $\mathrm{B}$ and pyrrométhène 597-C8) dissolved in two different solvents (water and $n$-decane, respectively). Figure 1 is extracted from Labergue et al. (2010): it depicts the evolutions of the fluorescence ratio of single calibrated droplets normalized by a reference taken in a cell, under isothermal conditions in the case of sulforhodamine $\mathrm{B}$ dissolved in water $\left(C=5 \times 10^{-6} \mathrm{~mol} \mathrm{l}^{-1}\right)$. A significant increase of the fluorescence ratio is observed when decreasing the droplet diameter, otherwise the normalized fluorescence ratio tends to 1 for the large droplets. To account for the effect of the droplet size on the fluorescence ratio, an empirical function $g_{12}(D)$ is added (Labergue et al. 2010):

$\frac{R_{12}}{R_{120}}=\frac{f_{12}(T)}{f_{12}(T 0)} g_{12}(D)$

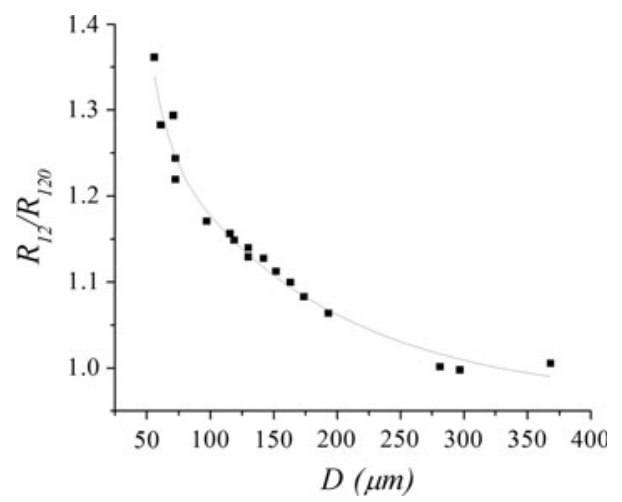

Fig. 1 Evolution of the normalized fluorescence ratio as a function of the diameter of calibrated single droplets at ambient temperature, $C=5 \times 10^{-6} \mathrm{~mol}^{-1}$ (Labergue et al. 2010)

\section{Experimental set-up and data processing}

\subsection{Experimental set-up: sprays facilities}

Two sprays, different in terms of droplet diameters and number concentrations, are tested. The first one, referred as spray (1), is a full cone spray generated by a Danfoss ${ }^{\circledR}$ swirled nozzle commonly used for burners. The injection pressure varies from 1 to 7 bars, and the liquid flow rate is of a few $1 / \mathrm{h}$. The second spray, referred as spray (2), is a flat spray generated by a Lechler ${ }^{\circledR}$ nozzle with an angle of $20^{\circ}$. Although the injection pressures range is the same as spray (1), the liquid flow rate is much higher (several $1 /$ min). Both sprays are injected vertically downwards, and the origin of the vertical axis ( $Z$ axis) is taken at the nozzle exit (Fig. 2). Spray (2) is mainly in a $X-Z$ plane and its thickness in the $Y$-direction is approximately $30 \mathrm{~mm}$.

\subsection{Measurement of the spray characteristics by PDA}

Sprays characteristics (droplet size and concentration) are evaluated with the use of a Phase Doppler Analyzer (PDA) manufactured by Dantec-dynamics ${ }^{\circledR}$ and equipped with a classic reception optics and a P80 signal processor. The laser excitation volume is formed using a LDA transmitter probe (Dantec-dynamics Fiber-Flow ${ }^{\circledR}$ probe). The laser source is an argon ion laser tuned at $\lambda=514.5 \mathrm{~nm}$. The receiver is positioned at a scattering angle $\varphi_{r}=45^{\circ}$, and the PDA is used in the refraction mode (Fig. 3). The focal length of the emission optics is changed when operating in sprays (1) or in (2) to account for the size range of these sprays that are very different. Consequently, the laser excitation volume is different for the two optical configurations as summarized in Table 1.

The PDA probe volume is defined by the projection of a slit (width $L_{p}=120 \mu \mathrm{m}$ ) on the laser excitation volume mentioned before. This corresponds to the intersection between the image of the slit in the focal plane of the PDA receiver and the laser excitation volume (Fig. 4). The image of the slit is about $257 \mu \mathrm{m}$ large whatever the configuration, since the reception focal length $f_{e}$ and the scattering angle are unchanged. Figure 5 presents two typical distributions of the droplet sizes, one corresponding to spray (1) and the other to spray (2). These distributions were measured at a different distance from the injector, given the fact that a longer distance is required for spray (2) to be fully atomized. A different injection pressure was also imposed, but additional measurements revealed that a change in the injection pressure has few effects on the droplet size distribution. The droplet size distributions are peaking at about $10 \mu \mathrm{m}$ for spray (1) and $75 \mu \mathrm{m}$ for spray (2). Moreover, it appears clearly that small droplets are more frequent in spray (1) than in spray (2). The mean 


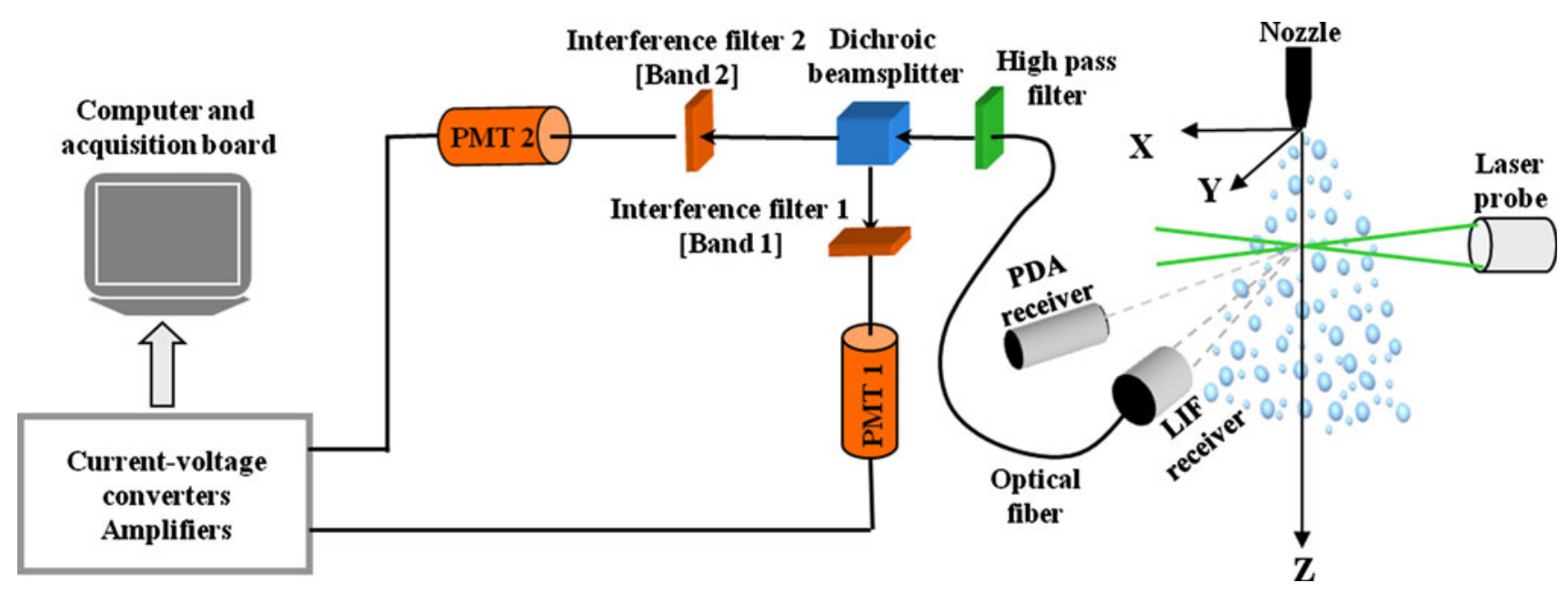

Fig. 2 Spray facilities and optical devices for 2cLIF detection

Fig. 3 Optical arrangement of LIF and PDA systems (top view)

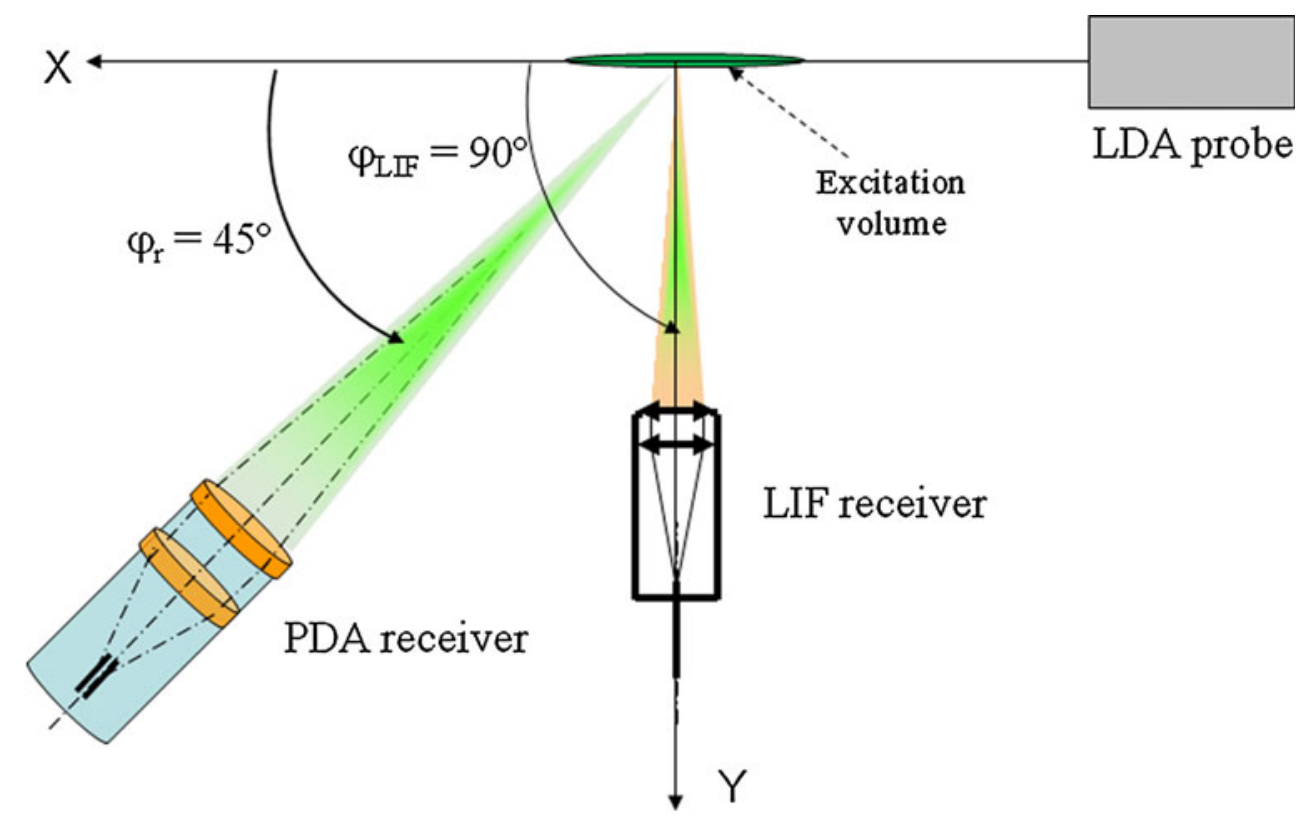

diameter $D_{10}$ is about $25 \mu \mathrm{m}$ for spray (1) and $200 \mu \mathrm{m}$ for spray (2). From Fig. 6, it can be clearly observed that the droplet number concentration $C_{d}$ of the spray (1) is widely higher than for the spray (2) and depends strongly on the injection pressure due to the increase of the liquid flowrate.

\subsection{LIF optical set-up and data processing}

The fluorescence signal is collected at right angle by an achromatic doublet coupled with an optical fiber (core diameter: $70 \mu \mathrm{m}$ ). The receiver consists in an achromatic doublet behind a changeable frontal lens. The LIF detection volume is defined by the intersection of the laser excitation volume and the projected image of the core of the optical fiber (Fig. 4). The diameter $d_{l}$ of this image varies with the focal length of the receiver front lens. It is, respectively, $d_{l}=210$ and $350 \mu \mathrm{m}$ for sprays (1) and (2) to account for the difference in size ranges of these sprays.

The collected signal comes through a high pass filter $\left(\right.$ Chroma $^{\circledR}$, HQ 522 LP) in order to remove the light scattered at the laser wavelength. The remaining fluorescence signal is split into the two spectral bands mentioned in Sect. 2.1 by means of a set of dichroic and interference filters (Fig. 2). The fluorescence signal is detected by means of two photomultiplier tubes and digitalized with a frequency of $5 \mathrm{MHz}$ for real-time processing. A threshold is fixed significantly above the noise level of the channels of detection. If a sequence has more than ten consecutive samples above this threshold, it is considered coming from 
Table 1 Characteristics of PDA optical configurations for sprays (1) and (2)

\begin{tabular}{lll}
\hline & Spray (1) & Spray (2) \\
\hline Optical configuration & & \\
Emission focal length, $\mathrm{f}_{e}(\mathrm{~mm})$ & 310 & 1,200 \\
Beam spacing, $\mathrm{b}_{s}(\mathrm{~mm})$ & 60 & 60 \\
Reception focal length, $\mathrm{f}_{r}(\mathrm{~mm})$ & 500 & 500 \\
Scattering angle angle, $\varphi_{r}\left({ }^{\circ}\right)$ & 45 & 45 \\
Maximum detectable droplet diameter, & 0.180 & 0.690 \\
$D_{\max }(\mathrm{mm})$ & & \\
Laser excitation volume size based on $1 / e^{2}(\mathrm{~mm})$ & \\
Size in $Z$ and $Y$ directions $\mathrm{a}_{z}=a_{y}$ & 0.152 & 0.582 \\
Size in $X$ direction $a_{x}$ & 1.562 & 23.3 \\
\hline
\end{tabular}

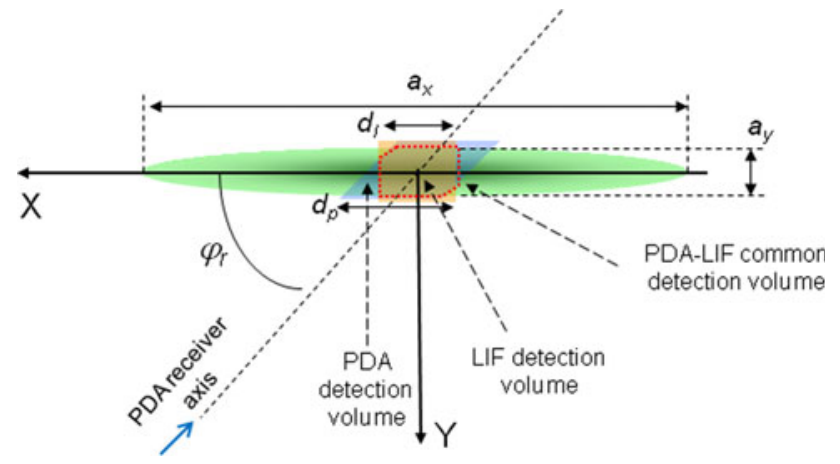

Fig. 4 Definitions of the LIF and PDA probe volumes

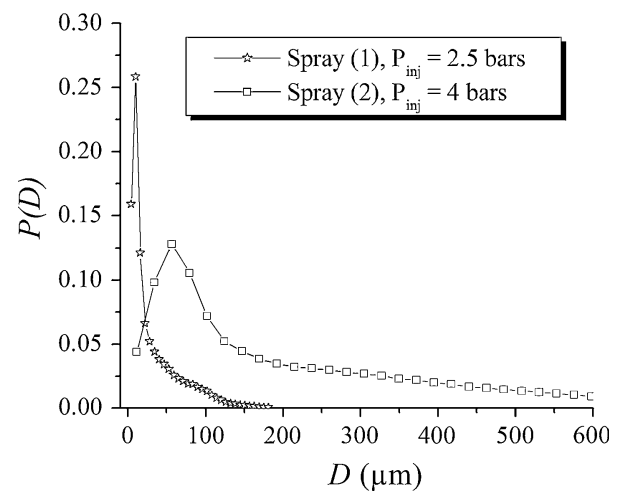

Fig. $5 \mathrm{PDF}$ of the droplet size distribution for measured at $Z=13 \mathrm{~mm}$ and $500 \mathrm{~mm}$, respectively, for sprays (1) and (2)

droplets. The fluorescence ratio $R_{12}$ is then calculated as follows

$R_{12}=\frac{I_{f 1}}{I_{f 2}}=\frac{\sum_{j=1}^{n} I_{f 1, j}-N_{1}}{\sum_{j=1}^{n} I_{f 2, j}-N_{2}}$

where $I_{f 1, j}$ and $I_{f 2, j}$ are, respectively, the fluorescence intensities integrated on the $j$ th droplet crossing the probe volume, for the spectral bands 1 and 2 , respectively. $n$ is

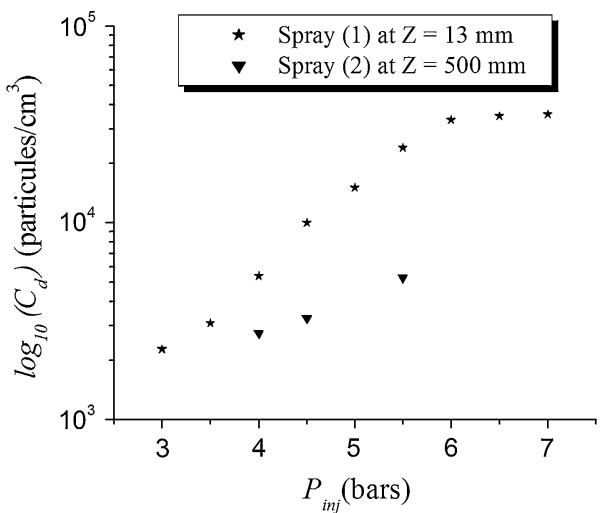

Fig. 6 Droplet number concentration ( $\log _{10}$ scale) measured at $Z=13$ and $500 \mathrm{~mm}$, respectively, for sprays (1) and (2)

the number of droplets detected during the acquisition period. $N_{1}$ and $N_{2}$ are the average dark noise values on both spectral bands.

\subsection{Combined LIF and PDA measurements in sprays}

In order to investigate the consequences of the droplet size effect on the 2 cLIF technique in polydisperse sprays, combined 2cLIF and PDA measurements have been performed. The measurement protocol and optical requirements are similar to those implemented by Depredurand et al. (2011). The combined 2cLIF/PDA system provides two data files recorded with the same time base, one corresponding to the droplet diameters measured by the PDA and the other one to the fluorescence intensities of the 2cLIF detection bands. Droplets detected in common by the PDA and 2cLIF techniques are identified on the basis of their arrival time and their transit time. Then, a fixed number of droplet size classes are defined, and the fluorescence intensity on each channels, $I_{f 1}$ and $I_{f 2}$, is averaged following Eq. 6. As a result, an average fluorescence ratio can be associated to a given droplet size class.

\subsection{Study of the droplet size range of the combined LIF/PDA measurements}

When a droplet crosses the probe volume, the collected luminous intensity is roughly proportional to $D^{2}$ for the PDA and to $D^{3}$ for LIF. When both techniques are combined, the wider dynamic range of the LIF signal limits the coupling of the techniques in terms of droplet sizes, which may influence the interpretations of the experimental results.

A rigorous determination of the detection range of the LIF measurement system should account for the droplets illumination by the Gaussian laser beams, the light focused by the curved surface of the droplets, and the limited field 
of view of the detection optics. Ray tracing is presently used to calculate the fluorescence signal. This method relies on geometrical optics and provides an approximate solution to Maxwell's equations that is valid as long as the light propagates through and around objects whose dimensions are much greater than the light wavelength. This approximation rules for droplet sizes larger than a few microns. Extensive calculations are carried out for droplet sizes ranging from 20 to $500 \mu \mathrm{m}$ in the case of the two aforementioned fluorescence excitation and detection systems. Laser beams are modeled as a bundle of parallel rays, the intensity of which follows an adequate Gaussian distribution in space. Each ray can be reflected and/or refracted by the surface of the droplet. Calculations are simplified by considering only rays of order 1, i.e., rays that do not undergo any internal reflection. Fresnel equations are used to determine the fraction of light that is transmitted through the droplet surface. In the absence of information on the temperature distribution within the droplet, the fluorescence is assumed to have everywhere the same coefficient of proportionality to the excitation intensity by the laser beams. The fluorescence photons are emitted isotropically, but only the photons arriving to the detector contributes to the measured signal. A second calculation is undertaken. It consists in shooting rays from the surface of the detector to the droplet. The fluorescence intensity is integrated along the ray paths inside the droplet. Here also, only first-order rays that are emerging parallel to the axis of the detection optics are considered. Calculations are limited to the case of droplets whose center coincides with the intersection between the laser beams and the optical axis of the detection optics.

If the concentration of the fluorescence is sufficiently low to neglect Beer's absorption, the emitted fluorescence intensity is directly proportional to the internal excitation field. This hypothesis is correct in the present case and remains valid, even if the droplet vaporizes significantly (Frackowiak and Tropea 2010). For example, for droplets of $100 \mu \mathrm{m}$ initial diameter, the error caused by absorption is on the order of $0.25 \%$. If the reduction of the diameter due to evaporation is roughly equal to $30 \%$ of the initial diameter, the error caused by the absorption will be on the order of $0.5 \%$. In such a case, the optical path decreases roughly proportionally to the droplet diameter $D$, as the dye concentration increases as the cubic diameter $D^{3}$. Figure 7 depicts the evolution of the collected LIF intensity for the two optical configurations as a function of the droplet diameter. It appears that the fluorescence intensity increases with the droplet size, until the droplet diameter is equal to the probe volume diameter. For larger droplets, the intensity has an almost constant value. When the droplet diameter is inferior to the probe volume diameter, the fluorescence intensity can be approximated by:

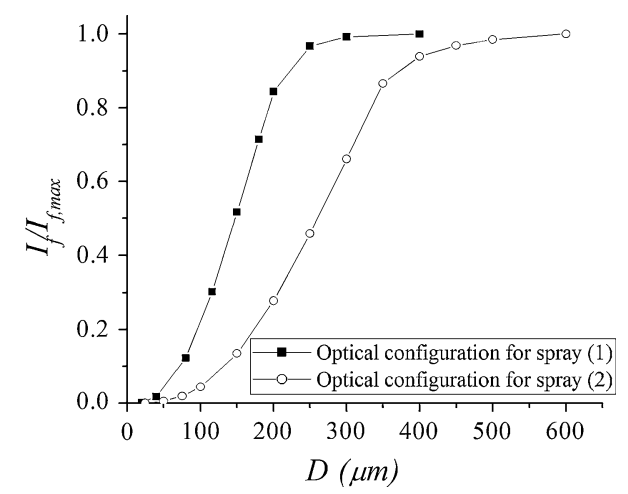

Fig. 7 Evolution of the LIF intensity as a function of the droplet diameter using ray tracing

$I_{f} \propto D^{k}$

where $k$ is an exponent that depends on the optical configuration. The values of $k$ for the two selected optical configurations are reported in Table 2. Furthermore, the exponent $k$ remains a constant for a given optical configuration, since the calculations are carried out under nonabsorbing conditions.

In the present experimental set-up, the fluorescence signal is acquired with a resolution of 4,096 levels (12 bits), and the detection threshold is about 100 levels, which corresponds roughly to the background noise. Thus, the dynamic range of the signal is approximately 40. From a practical point of view, $D_{\min }$ and $D_{\max }$ are fixed by the high voltages of the LIF photodetectors. The high voltages are adjusted in order to optimize the detection of the smallest droplets. However, increasing the high voltage leads to a risk of photodetectors saturation that switch off the acquisition. Therefore, a trade-off has generally to be found with missing droplets among the smallest and a cut-off among the largest. Figure 8 presents typical evolutions of the normalized fluorescence intensity collected by the detectors as a function of the droplet diameter on the second spectral band for both spray (1) and in Fig. 9 for spray (2). For both cases, the cut-off corresponding to $D_{\text {min }}$ can be read directly on the plots and knowing $k, D_{\max }$ can be derived from Eq. 7:

$D_{\max } \propto 40^{1 / k} D_{\min }$

Table 2 LIF size range detection for both sprays

\begin{tabular}{lcl}
\hline & $\begin{array}{l}\text { Spray (1) optical } \\
\text { configuration (1) }\end{array}$ & $\begin{array}{l}\text { Spray (2) optical } \\
\text { configuration (2) }\end{array}$ \\
\hline$k$ & 2.81 & 2.74 \\
$D_{\max }(\mu \mathrm{m})$ & 104 & 385 \\
$D_{\min }(\mu \mathrm{m})$ & 28 & 100 \\
\hline
\end{tabular}


In the following, droplets having a size above $D_{\max }$ are not retained in the analysis of the results. Values of $D_{\min }$ and $D_{\max }$ are reported in Table 2 for both sprays.

\section{Experimental results}

\subsection{Case of spray (1)}

\subsubsection{Influence of the depth of field}

Figure 10 depicts the evolution of the normalized ratio $R_{12} / R_{120}$ as a function of the droplet diameter $D$ in the spray centerline $(X=Y=0)$ at $Z=13 \mathrm{~mm}$, for three values of the injection pressure $\left(R_{120}\right.$ being the fluorescence ratio in a cell at room temperature). Furthermore, the data obtained for calibrated droplets in monodisperse streams by Labergue et al. (2010) are superimposed. All the experiments were carried out with the same injection temperature that corresponds also to the room temperature. For the lower injection pressure $\left(P_{\mathrm{inj}}=2.5 \mathrm{bars}\right)$, the normalized fluorescence ratio is comparable to its values for monodisperse droplets. The effect of the droplet on the fluorescence ratio already highlighted for monodisperse droplets is also observed in the sprays. For higher values of the injection pressure, the curves are shifted toward higher $R_{12} / R_{120}$ values.

Figure 11 presents some pictures of the fluorescence light emitted by the droplets for different injection pressures. Above 2.5 bars, a halo of light can be seen around the laser beams. This halo indicates that the laser light is scattered in the spray in such an extent that even droplets far from the laser beams can emit a visible amount of fluorescence. Laser light scattering tends to increase with the droplet number concentration, and hence the injection pressure as presented in Fig. 6. Given the large depth of

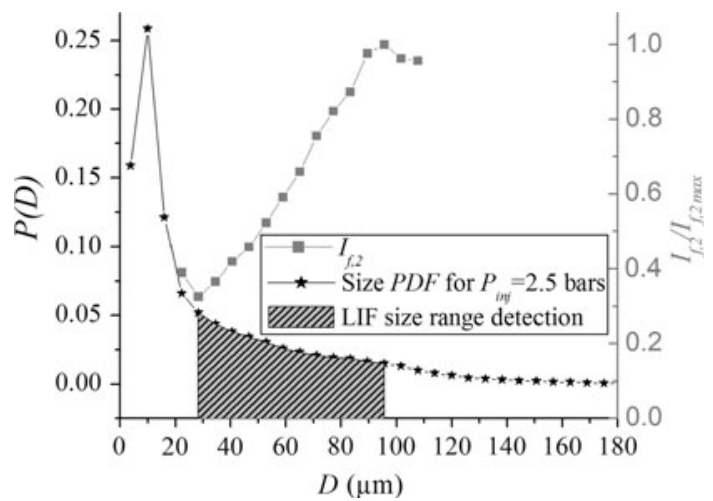

Fig. 8 Size range dynamic of LIF technique superimposed on the droplet size distribution for the spray (1) with $P_{\text {inj }}=2.5$ bars. Evolution of the fluorescence intensity collected on the second spectral band as a function of the droplet diameter

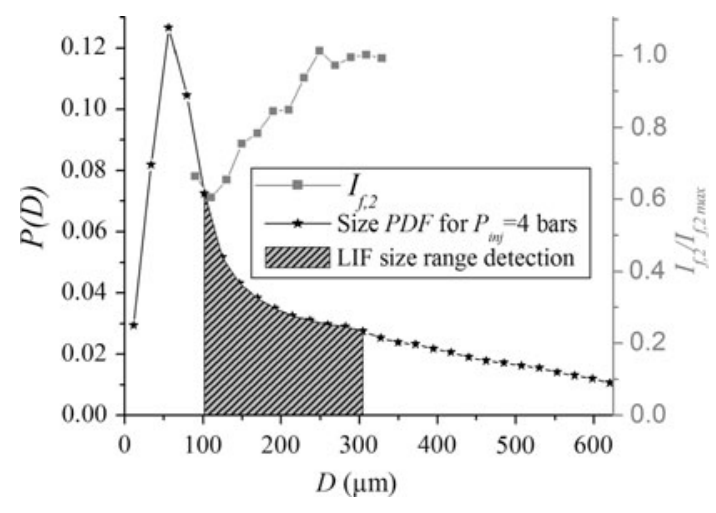

Fig. 9 Size range dynamic of LIF technique superimposed on the droplet size distribution for the spray (2) with $P_{\text {inj }}=4$ bars. Evolution of the fluorescence intensity collected on the second spectral band as a function of the droplet diameter

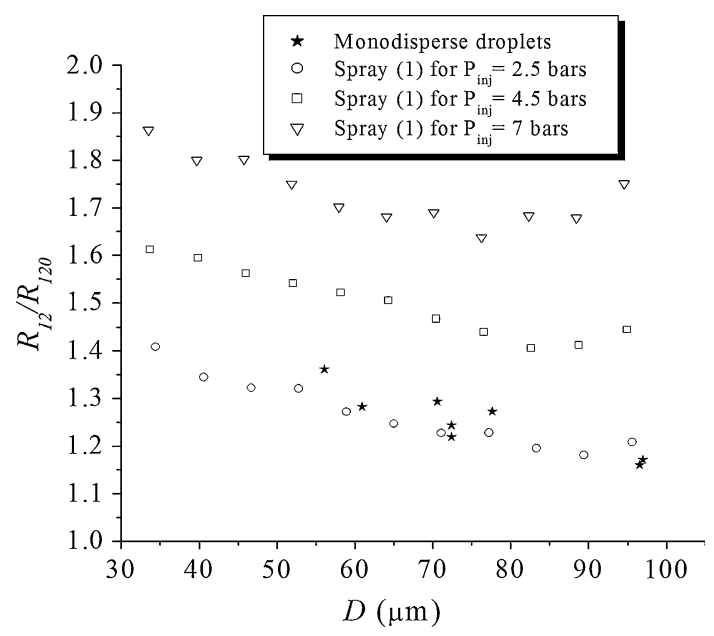

Fig. 10 Results of 2cLIF/PDA combined measurements: evolution of the normalized fluorescence ratio as a function of the droplet diameter for three injection pressures at $Z=13 \mathrm{~mm}$. $R_{120}$ is the fluorescence ratio measured in a cell at the same temperature

field of the achromatic doublet in use, it is likely that the fluorescence coming from out-of-field droplets contributes to the fluorescence signal. Out-of-field fluorescence is almost absent for $P_{\text {inj }}=2.5$ bars, but increasingly present for higher pressures, corresponding to higher spray densities.

Figure 12 shows a sample of the fluorescence signal $\left(I_{f 1}\right.$ and $I_{f 2}$ ) over a time interval of $2 \mathrm{~ms}$. In this figure, it can be observed that the signal returns to the background noise in the absence of droplets. This supports the assumption that the out-of-field fluorescence affects the signal only when a droplet crosses the probe volume. According to the droplet size distribution (Fig. 5), the main part of the droplets is of small size (65\% are $<30 \mu \mathrm{m}$ in diameter). When a droplet is detected and validated, the out-of-field fluorescence captured by the collection system is mainly due to an ensemble of small droplets (typically, the most probable 

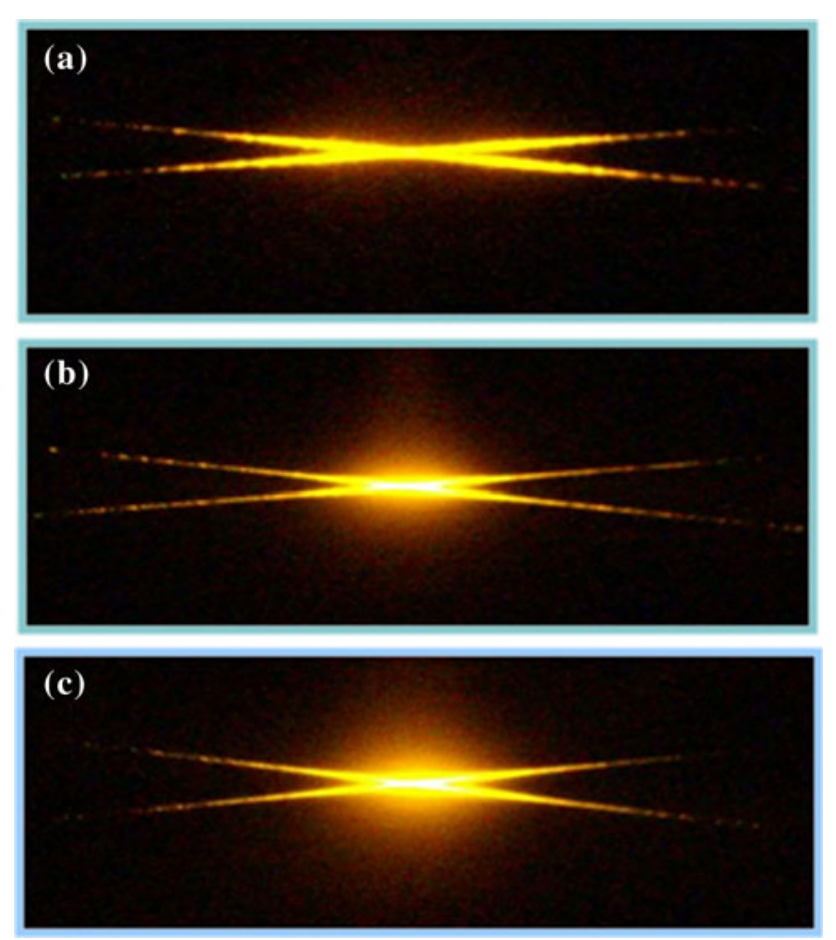

Fig. 11 Visualization of the fluorescence in the vicinity of the excitation probe volume for $P_{\text {inj }}=2.5$ bars $\mathbf{a}, P_{\text {inj }}=4.5$ bars $\mathbf{b}$ and $P_{\text {inj }}=7$ bars c

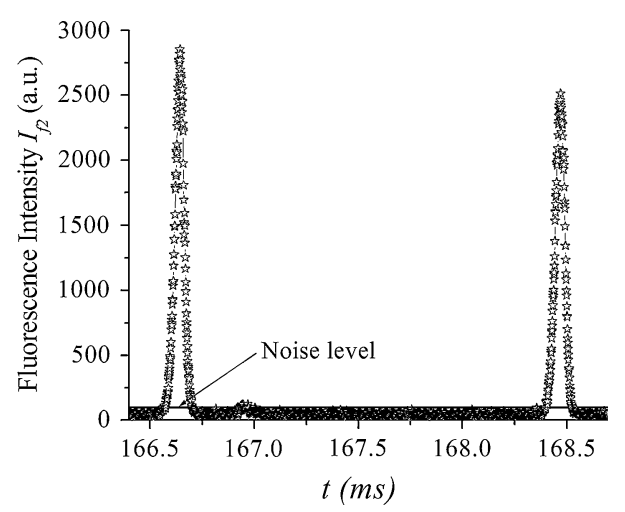

Fig. 12 Typical temporal fluorescence signal detected on spectral band 2 recorded in the spray (1)

are droplets below $10 \mu \mathrm{m}$ ), the fluorescence of which is strongly affected by the droplet size. This provides an explanation for the increase in the fluorescence ratio with the injection pressure, since the fluorescence ratio is increased for small droplets as presented in Fig. 1.

\subsubsection{Reduction of the depth of field}

The depth of field of the collection is reduced with the used of a long distance microscope QM-100 from Questar ${ }^{\circledR}$ coupled with an optical fiber. A fluorescent spot generated by the illumination of a monodisperse droplet stream $(D \approx 130 \mu \mathrm{m})$ seeded by sulforhodamine B is used to quantify the depth of field of the detection system. The LIF collection optics (either the long distance microscope or the achromatic lens doublet) is progressively shifted along the $Y$-axis, and the evolution of the fluorescence signal is plotted as a function of the $Y$ distance (Fig. 13). It appears clearly that the intensity collected by the long distance microscope decreases very quickly when the distance from the focal plane is increased: $5 \%$ of the maximum intensity remains after a displacement of $2.8 \mathrm{~mm}$ for the long distance microscope compared to $70 \%$ for the achromatic doublet. The fact that a similar evolution is observed for $I_{f 1}$ and $I_{f 2}$ confirms that the optical devices are adequately achromatic.

The experiments performed with the achromatic doublet in Sect. 4.1 are repeated with the long distance microscope. The results are reported in Fig. 14 and compared with those obtained with the achromatic doublet. For $P_{\text {inj }}=2.5$ bars, the fluorescence ratio obtained with the achromatic doublet and the long distance microscope are overlapping. For higher injection pressures (i.e., $P_{\mathrm{inj}}=4.5$ and 7 bars), the shift in the fluorescence ratio is considerably reduced with the use of the long distance microscope, but it does not completely disappear, especially at 7 bars. The droplet concentration still remains too high.

From this study of spray (1), it can be concluded that temperature/size correlations can be seriously biased when the collection optics of the 2cLIF has a large depth of field given the effect of the droplet size on the fluorescence spectrum. This problem is on the increase of the fluorescence ratio when droplets of small size are in a large number concentration. Finally, decreasing the depth of field of the collection reduces this bias. However, in the present case, the spray density is too high to eliminate totally the problem for the higher injection pressures. In the following section, experiments carried out in a spray having a lower droplet number concentration will be presented.

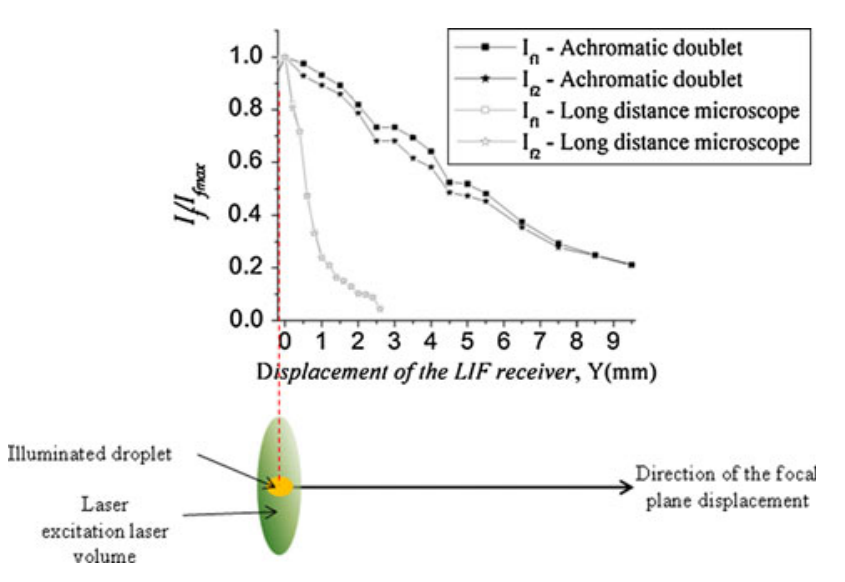

Fig. 13 Evolution of the fluorescence intensities detected on the 2 cLIF spectral bands as a function of the displacement of the focal plane for both collection optics devices 


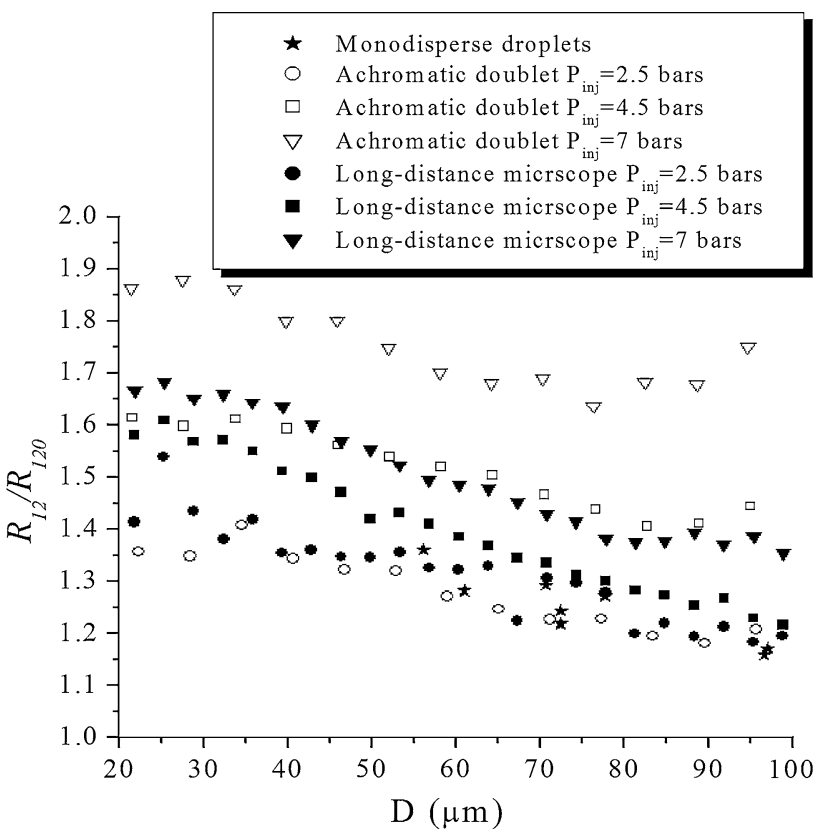

Fig. 14 Combined 2cLIF/PDA results obtained with spray (1) and by using the long distance microscope for three injection pressures at $z=13 \mathrm{~mm} . R_{120}$ is the fluorescence ratio measured in a cell at the same temperature

\subsection{Case of spray (2)}

Spray (2) has a wider size distribution and a much lower droplet concentration than spray (1) (Fig. 6). Combined LIF/PDA measurements are reported in Fig. 15, for two injection pressures, $P_{\text {inj }}=4$ bars and $P_{\text {inj }}=6$ bars. To help the comparisons, data related to spray (1) and monodisperse lines of droplets have been also superimposed. Whatever the injection pressure, the normalized fluorescence ratios $R_{12} / R_{120}$ are on the same curve as the data of spray (1) at $P_{\mathrm{inj}}=2.5$ bars and the data of the monodisperse droplets. This behavior is directly linked to the lower density of spray (2) that limits the laser scattering and consequently, the out-of-field fluorescence. Furthermore, almost half of the droplets in spray (2) are larger than $100 \mu \mathrm{m}$, and beyond this diameter, it can be considered that the droplet size has almost no influence on the fluorescence ratio (Fig. 1).

\section{Intrinsic correction of the effect of the droplet size on the fluorescence ratio}

\subsection{Three-color LIF measurements}

In Sect. 2.2, the function $g_{12}(D)$ was introduced to take into account the effect of the droplet size on the fluorescence ratio (Eq. 5). This function would allow correcting this effect, but is a priori unknown and has to be determined

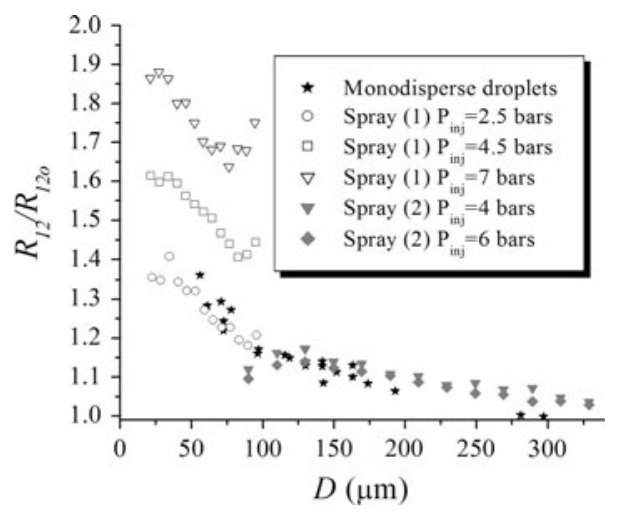

Fig. 15 Results of LIF/PDA combined measurements for spray (2) at $\mathrm{Z}=500 \mathrm{~mm}$ and comparison the spray (1). $R_{120}$ is the fluorescence ratio measured in a cell at the same temperature

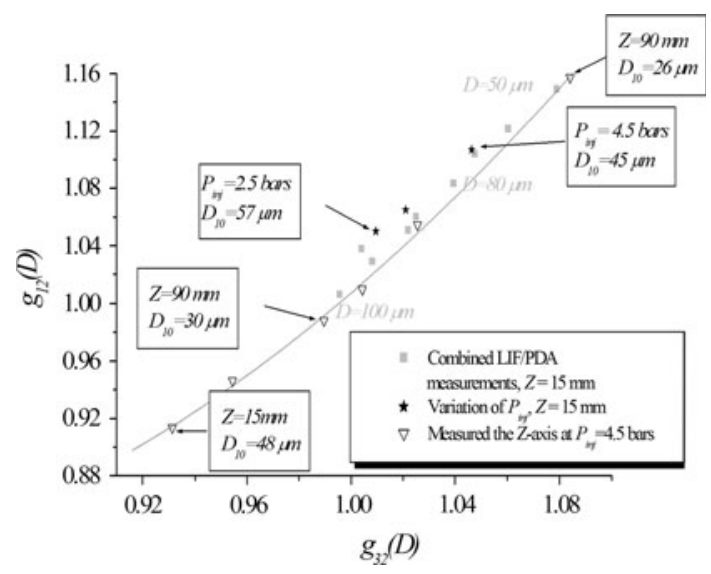

Fig. 16 Relationship between $g_{12}$ and $g_{32}$ under isothermal condition in the case of spray (1) at room temperature

empirically. An important issue is also to achieve an intrinsic correction for this effect, i.e., a method able to measure the temperature without the requirement to measure simultaneously the droplet size by PDA. The following paragraph describes an empirical procedure for such an intrinsic correction.

For this intrinsic correction, a third spectral band [555; $575 \mathrm{~nm}]$ is selected. This band is particularly sensitive to the spectral influence of the droplet size. A second fluorescence ratio is derived from the fluorescence intensities measured on that band and the second band:

$\frac{R_{32}}{R_{320}}=\frac{f_{32}(T)}{f_{32}\left(T_{0}\right)} g_{32}(D)$

where $f_{32}(T)=f_{3}(T)-f_{2}(T)$ is determined experimentally by a calibration $\left(a_{32}=212,000 \mathrm{~K}^{2}\right.$ and $\left.b_{32}=-1,315 \mathrm{~K}\right)$, and the reference $R_{320}$ is taken in a cell at the temperature $T_{0}$. This ratio presents a particularly low sensitivity to the temperature $\left(<0.1 \% /{ }^{\circ} \mathrm{C}\right)$. For that reason, in the further uncertainties evaluation, the potential uncertainties on the 
calibration procedure of $R_{32}$ will not be considered. $g_{12}(D)$ and $g_{32}(D)$ are unique functions depending only the spectral bands of detection and the fluorescent tracer. They can be determined experimentally by measuring $R_{12} / R_{120}$ and $R_{32} / R_{320}$ for several droplet diameters under isothermal conditions (i.e., all droplets assumed at the same temperature $T$ everywhere in the spray), while $R_{120}$ and $R_{320}$ are obtained separately in a cell.

Combined PDA/2cLIF measurements can be used to determine the normalized fluorescence ratios $R_{12} / R_{120}$ and $R_{32} / R_{320}$ per diameter class. However, a method not relying on the coupling between PDA and 2cLIF techniques has been also tested. In this method, the mean droplet diameter $D_{10}$ is characterized using PDA, while mean values of the fluorescence ratios $R_{12}$ and $R_{32}$ are measured simultaneously. The measurements are repeated for several injection pressures to make $D_{10}$ vary from 30 to $90 \mu \mathrm{m}$.

For both approaches, the measurements are performed at ambient temperature and sufficiently close to the atomizer exit, to consider as reliable the temperature provided by a thermocouple placed in the immediate vicinity of the laser excitation volume. Then, the quantities $f_{12}(T) / f_{120}\left(T_{0}\right)$ and $f_{32}(T) / f_{320}\left(T_{0}\right)$ corresponding, respectively, to $g_{12}(D)$ and $g_{32}(D)$ are determined. Figure 16 presents the evolution of $g_{12}$ as a function of $g_{32}$. However, whatever the method, the plot $g_{12}$ as a function of $g_{32}$ is collapsing. It follows a monotonous evolution that can be interpolated by a secondorder polynomial:

$g_{12}=\alpha g_{32}^{2}+\beta g_{32}+\gamma$

Finally, combining Eqs. 5, 9 and 10 leads to:

$\frac{R_{12}}{R_{120}} \frac{f_{12}(T)}{f_{12}\left(T_{0}\right)}=\alpha\left[\frac{f_{32}\left(T_{0}\right)}{f_{32}(T)} \frac{R_{32}}{R_{320}}\right]^{2}+\beta\left[\frac{f_{32}\left(T_{0}\right)}{f_{32}(T)} \frac{R_{32}}{R_{320}}\right]+\gamma$

Knowing $\alpha, \beta$, and $\gamma$, Eq. 11 can be solved to determine the temperature from the measurements of $R_{12}$ and $R_{32}$.

\subsection{Measurements in a heated spray}

Temperature measurements have been performed in spray (1), for an injection temperature higher than the ambient temperature $\left(T_{\mathrm{inj}}=51^{\circ} \mathrm{C}\right)$ and for an injection pressure of $P_{\text {inj }}=4.5$ bars. The fluorescence is collected by using the long distance microscope to limit the depth of field.

Combined 2cLIF/PDA measurements are performed at several locations along the spray axis. The variation of the temperature $T$ is presented in Fig. 17 as a function of the droplet diameter for different downstream locations, $Z=20,50$, and $90 \mathrm{~mm}$. As expected, it can be observed that the cooling is more pronounced for the smallest droplets than for the largest ones. The mean droplet temperature $T_{m}$ is also determined by processing the fluorescence ratios by Eq. 6 . The evolution of this temperature is displayed as a function of the axial distance $Z$ in Fig. 18 . A decrease of about $22^{\circ} \mathrm{C}$ is observed when the droplets cover a distance of $90 \mathrm{~mm}$. These mean temperatures can be also compared to the combined 2cLIF/PDA measurements from which a mean droplets temperature can be derived as followed:

$T_{m}=\frac{\sum_{j=1}^{N_{c}} D_{j}^{k} N_{j} T_{j}}{\sum_{j=1}^{N} D_{j}^{k} N_{j}}$

where $j$ is the droplet size class index, $N_{c}$ the number of size classes, $N_{j}$ and number of droplets per size class and $T_{j}$ of temperature corresponding to the $j$ th class. The parameter $k$ allows accounting for the dependence of the fluorescence intensity on the droplet diameter (Eq. 7). $T_{m}$ is also reported in Fig. 18. In addition, the temperature evolution for two diameter classes, $D=56$ and $110 \mu \mathrm{m}$, is presented. It appears that this second form of $T_{m}$ follows an evolution similar to the temperature obtained using Eq. 6. However, it is systematically higher. This is probably linked to the fact that mean droplet temperatures obtained by combined 2cLIF/PDA are also filtered by the specific PDA validation criteria (phase matching with the two pairs of detectors, slit and trajectory ambiguities, etc....).

\subsection{Evaluation of the uncertainties}

The main error source can be found in the measurement of the fluorescence ratio and in the temperature calibration of $R_{12}$ (see Sect. 2.1). The accuracy of the measurement of this parameter is directly related to the number of samples used to calculate the fluorescence signal mean value on each of the spectral bands, $I_{f 1}$ and $I_{f 2}$.

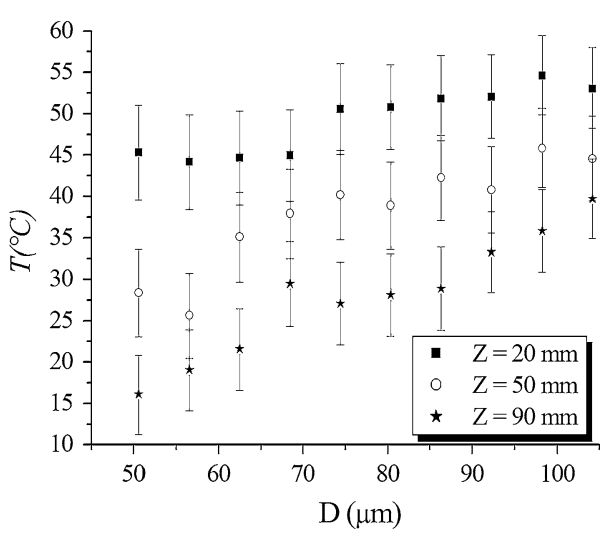

Fig. 17 Evolution of the droplet temperature as a function of the droplet diameter at three downstream locations in the case of the spray (1) for $P_{\mathrm{inj}}=4.5$ bars and $T_{\mathrm{inj}}=51^{\circ} \mathrm{C}$ 


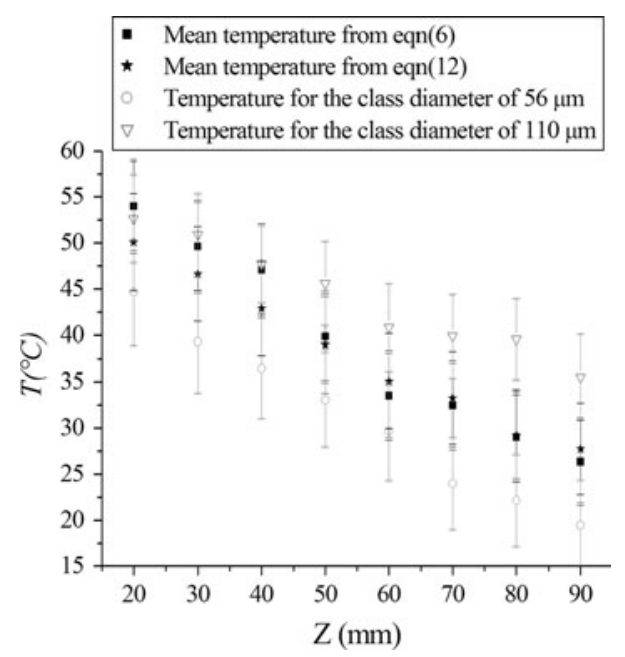

Fig. 18 Evolution of the mean temperature along the $Z$-axis in the case of the spray (1) for $P_{\text {inj }}=4.5$ bars and $T_{\text {inj }}=51^{\circ} \mathrm{C}$. Comparison with the mean temperature derived from Eq. 6

The number of samples is adjusted so as to obtain a stochastic error on the mean value of the fluorescence intensity inferior to $0.5 \%$, regardless the size class. As a consequence, the stochastic error on the fluorescence ratio is on the order of $1 \%( \pm 0.5 \%)$, which propagates in the different equations that relate the fluorescence ratio and the measured temperature (Eqs. 10, 11).

Then, the maximum error can be determined by propagating the error on the measurements of the fluorescence ratios and the temperature sensitivity coefficients in Eqs. 10 and 11. The error bars for the worst case are reported in Figs. 17 and 18.

\section{Conclusions}

This paper has presented some improvements for the measurement of the droplet temperature in sprays using two-color LIF thermometry, and the reliability of the technique is assessed in two sprays having significantly different droplet sizes and number concentration. Combined LIF and PDA measurements highlight the influence of the effect of the droplet size, in particular when the depth of field of the fluorescence receiving optics is so large that an out-of-field fluorescence due to multiple scattering of the laser light by the droplet can contribute to the signal. The risk of errors is mainly due to the presence of small droplets when a dense spray is involved. In the case of a dense spray, a long distance microscope can be used to decrease the depth of field and then significantly remove the influence of the out-of-field fluorescence. Moreover, an empirical method to correct the residual effect of the droplet size on the fluorescence was proposed.
It implies to add a third spectral band of detection, for which the sensitivity to temperature is low and the sensitivity to size effect is high. Finally, combined LIF and PDA measurements are applied on a quite dense overheated spray of water. As expected, a decrease of the droplet temperature is observed along the main axis of the spray, and this decrease is all the more important that the droplet size is small.

In the future works, it is planed to compare the 3cLIF measurements developed in the present paper to GRT, developed by the team of CORIA, in Rouen (France).

Acknowledgments This work has been conducted in the framework of the ASTRA program supported by CNRS and ONERA.

\section{References}

Bernardin JD, Stebbins CJ, Mudawar I (1997) Mapping of impact and heat transfer regimes of water drops impinging on a polished surface. Int J Heat Mass Transf 40:247-267

Castanet G, Lavieille P, Lebouche M, Lemoine F (2003) Measurement of the temperature distribution within monodisperse combusting droplets in linear streams using two-color laserinduced fluorescence. Exp Fluids 35:563-571

Castanet G, Lienard T, Lemoine F (2009) Dynamics and temperature of droplets impacting onto a heated wall. Int J Heat Mass Transf 52:670-679

Depredurand V, Miron P, Labergue A, Wolff M, Lemoine F (2008) A temperature-sensitive tracer suitable for two-color laser-induced fluorescence thermometry applied to evaporating fuel droplets. Meas Sci Technol 19:1-12

Depredurand V, Delconte A, Lemoine F (2011) Combined PDA and LIF applied to size-temperature correlations measurements in heated spray. Exp Fluids 50:561-571

Frackowiak B, Tropea C (2010) Fluorescence modeling of droplets intersecting a focused laser beam. Appl Opt 35:1386-1388

Gossage HE, Melton LA (1987) Fluorescence thermometers using intermolecular exciplex. Appl Opt 26:2256-2259

Habuchi S, Kim HB, Kitamura N (2000) Chemical size-effect on fluorescence lifetime of rhodamine $6 \mathrm{G}$ in ethylene glycol/water microdroplets in polydimethylsiloxane matrix. J Photochem Photobiol 133:189-196

Kim HJ, Kihm KD, Allen JS (2003) Examination of a ratiometric laser induced fluorescence thermometry for microscale spatial measurement resolution. Int J Heat Mass Transf 46:39673974

Labergue A, Depredurand V, Delconte A, Castanet G, Lemoine F (2010) New insight into two-color LIF thermometry applied to temperature measurements of droplets. Exp Fluids 49:547-556

Lavieille P, Lemoine F, Lebouché M, Lavergne G (2001) Evaporating and combusting droplet temperature measurements using twocolor laser-induced fluorescence. Exp Fluids 31:45-55

Maqua C, Castanet G, Grisch F, Lemoine F, Kristiadi T, Sazhin SS (2008) Monodisperse droplet heating and evaporation: experimental study and modeling. Int J Heat Mass Transf 51:39323945

Murray MA, Melton LA (1985) Fluorescence methods for determination of temperature in fuel spray. Appl Opt 24:2783-2787

Sakakibara J, Adrian RJ (1999) Whole field measurement of temperature in water using two-color laser induced fluorescence. Exp Fluids 26:7-15 
Sakakibara J, Hishida K, Maeda M (1997) Vortex structure and heat transfer in the stagnation region of an impinging plane jet. Int $\mathrm{J}$ Heat Mass Transf 40:3163-3176

Sazhin SS (2006) Advanced models for fuel droplets heating and evaporation. Prog Energy Combust Sci Energy 32:162-214

Van Beek J, Grosges T, De Giorgi MG (2003) Global rainbow thermometry assessed by airy and Lonrenz-Mie theories and compared with phase Doppler anemometry. Appl Opt 42:40164022

Vetrano MR, Gauthier S, Beek J, Boulet P, Buchlin JM (2006) Characterization of a non-thermal water spray by global rainbow thermometry. Exp Fluids 40:15-22 